« Ah sourdra-t-il de la bataille une mélodie à la taille immense de nos horizons »

Aragon, «Pour un chant national », Les Yeux d'Elsa, v. 36

\title{
Louis Aragon et le jeu médiéval
}

Dans l'œuvre de Louis Aragon, la période de la Résistance marque un important renouveau des formes et de l'inspiration poétiques. Durant l'avant-guerre, il a relu nombre d'œuvres médiévales et cela a confirmé en lui une fascination déjà ancienne pour les troubadours ${ }^{1}$. La poésie médiévale devient alors essentielle pour un poète qui aspire tout à la fois à renouveler le langage poétique, donner une expression à un amour et exalter le courage national. Certes, aucun recueil n'est fondé sur la seule inspiration médiévale, mais les œuvres résistantes d'Aragon (Le Crève-cœur (1940), Les Yeux d'Elsa (1942), Brocéliande (1942), En Français dans le texte (1943), Le Musée Grévin (1943), et La Diane française $(1945)^{2}$ ) sont toutes marquées par l'importance des motifs médiévaux et par l'emprunt de formes préclassiques, visant à déstabiliser les conventions poétiques contemporaines.

Cette insertion du Moyen Âge dans une poésie tournée vers l'événement et vers l'action peut paraître paradoxale. Mais le jeu avec le Moyen Âge vient en fait servir la perspective résistante, en permettant un décalage favorable à l'expression engagée, en offrant un fond légendaire et en suscitant un renouvellement poétique original. Mais, si Aragon joue sur la référence médiévale, c'est pour inciter à une action spécifique sur le monde réel.

\section{Un patronage explicite}

Le Moyen Âge hante autant les poèmes d'Aragon que les textes théoriques qui, souvent, les accompagnent. Face à la propagande nazie qui, elle aussi, emploie des références médiévales, la poésie d'Aragon tente de lancer une contre-mythologie politique.

Sont convoqués dans les poèmes ou les essais les troubadours suivants: Arnaut Daniel, Bertran de Born, Peire Cardenal et Peire Vidal. Ils servent de référence identificatoire, comme en témoigne le début de l'essai Les Poissons noirs, ou de la réalité en poésie :

${ }^{1}$ Dans sa biographie, Aragon, une vie à changer (Paris, Flammarion, 1994, p. 378), Pierre Daix écrit : Qu'Aragon ait recueilli et lu entre 1937 et 1939 tout ce qu'il pouvait trouver d'une poésie française des origines qui, le plus souvent, n'avait plus été rééditée depuis le XIX $\mathrm{e}^{\mathrm{e}}$ siècle, déchiffrant chansons de geste, romans courtois, poèmes lyriques des troubadours et des trouvères, voilà qui peut sembler aussi prodigieux que la rencontre de Picasso, au début de 1906, avec l'exposition de sculpture ibérique au Louvre.

Voir à ce sujet Elodie Burle et Valérie Naudet, « Nager à contre-courant (une approche du sujet lyrique entre mesure et démesure à partir de quelques poèmes d'Arnaut Daniel et Louis Aragon) », Revue des langues romanes, t. CX, 2006, $\mathrm{n}^{\circ} 2$, p. 507-525; Philippe Gardy, «Aragon lecteur des troubadours : emprunt et déplacement d'un mythe », Mythes, Images, Représentations, Trames, Limoges, Didier, p. 269-277; Roger Lassalle, «La poésie médiévale dans l'œuvre critique et l'œuvre poétique d'Aragon », L'Image du Moyen Âge dans la littérature française de la Renaissance au XX siècle, Poitiers, La Licorne, 1982, t. 1, p. 195-211.

${ }^{2}$ Les éditions originales sont les suivantes : Le Crève-cour, Paris, Gallimard, 1941; Les Yeux d'Elsa, Neuchâtel, Cahiers du Rhône, 1942 ; Brocéliande, Neuchâtel, La Baconnière, 1942 ; Le Musée Grévin, Paris, Éditions de Minuit, 1943 ; La Diane française, Paris, Seghers, 1944.

Sauf indication contraire, nous renvoyons dans cet article à l'édition récente des œuvres poétiques complètes : Louis Aragon, Euvres poétiques complètes, sous la direction d'Olivier Barbarant, Bibliothèque de la Pléiade, Paris, Gallimard, 2007 [OPC]. 

Vidal :

Je viens de relire le Musée Grévin, et bonne envie me prend d'en parler comme Peire

Una chanso ai faita mortalmen

Si qu'eu no sai dire com ni consi

Qu'anc noit ni jorn, de ser ni de mati,

Non tenc mon cor ni nulh mon pensamen ${ }^{3}$

La citation médiévale est liée à l'amour comme à l'idée de double sens, puisque Peire Vidal, comme tous les troubadours, a chanté une dame sous un senhal, un pseudonyme permettant aux seuls initiés de reconnaître la personne concernée. Placée au début du recueil de résistance le plus violemment satirique d'Aragon, cette clef fait un lien indiscutable entre amour courtois et engagement.

Le patronage des poètes lyriques s'introduit jusque dans le corps des poèmes. Dans «Richard Cœur-de-Lion »(Les Yeux d'Elsa), Aragon évoque Blondel de Nesle, l'un des premiers trouvères courtois, favori de Richard Cœur-de-Lion, qui selon la légende aurait permis par son ingéniosité de libérer le roi emprisonné : il aurait réussi à trouver le château où ce dernier était enfermé en chantant une romance qu'ils avaient composée ensemble. Cette libération aurait donc été suscitée par l'usage très concret d'un poème ${ }^{4}$. Sous le couvert de cette figure historique, la dernière strophe du poème d'Aragon constitue un appel à peine caché à la Résistance :

Tous les Français ressemblent à Blondel

Quel que soit le nom dont nous l'appelions

La liberté comme un bruissement d'ailes

Répond au chant de Richard Cœur-de-Lion ${ }^{5}$

La référence aux poètes lyriques fait ainsi partie du processus démonstratif du poème de résistance. Un parallèle entre troubadour et poète résistant est d'ailleurs établi dans « Pour un chant national » (Les Yeux d'Elsa). À la faveur d'un jeu de mots inspiré par une quasi-homonymie, Aragon établit une comparaison entre Alain Borne, auteur de Neige et 20 poèmes, dont il trouve la poésie trop peu engagée, et Bertran de Born, célèbre pour l'acuité politique de ses sirventes - on lui a même attribué un rôle dans les dissensions entre Henri II d'Angleterre et Richard Cœur-de-Lion :

Vous me faites penser à ce poète qui s'appelait Bertrand de Born presque comme vous

Alain Borne un pays sans borne

Ressemble à votre poésie

Où des demoiselles choisies

Comme au beau temps de l'unicorne

Attendent un Bertrand de Born songez-y $y^{6}$

Qui leur chante les raisons de vivre et d'aimer les raisons d'aimer et d'en mourir

Ce poème aux strophes hétérométriques et clos par le retour de la première strophe à la fin s'inspire de la forme du rondeau médiéval. Le lien entre chant d'amour et poème engagé est rappelé dans les lignes en prose évoquant, dans un chiasme, le double engagement amoureux et politique. La fonction du poète est ainsi illustrée par la référence à un modèle médiéval.

\footnotetext{
${ }^{3}$ OPC, p. 913 ; la traduction suivante y est donnée :

Une chanson - j'ai faite triste à en mourir

Tant que je ne sais dire comme ni comment,

Car nuit ni jour, le soir ni le matin,

Je ne maîtrise mon cœur ni aucune de mes pensées.

${ }^{4}$ Il s'y ajoute une identification du locuteur au roi emprisonné, puisque l'écriture de ce poème date d'une période où Aragon et Elsa Triolet étaient eux aussi prisonniers.

${ }^{5}$ Id., p. 781, v. 33-36.

${ }^{6}$ Ibid., v.7-12.
} 


\section{Le Moyen Âge comme comparant : le jeu de la référence historique}

Aragon utilise souvent le Moyen Âge comme comparant pour la période contemporaine. Les croisades surgissent dans le poème « Marche française » de La Diane française, mais il s'agit désormais d'une croisade à l'intérieur des frontières :

Croisés non pour une aventure

Une lointaine sépulture

Mais pour le pays envahi

Contre l'envahisseur haï ${ }^{7}$

Et quand l'époque des croisades n'est pas utilisée comme aune pour évaluer l'époque actuelle, elle est directement décrite, mais en induisant un sens sous-jacent qui fait allusion au combat résistant. Dans «Les Croisés » (Le Crève-cœur), le but premier de la croisade est la Terre Sainte (sous laquelle on devine la France), mais le but réel est la femme aimée, en l'occurrence Éléonore d'Aquitaine, qui représente en fait la figure allégorique de la Liberté :

\section{Mais ce ne fut enfin que dans quelque Syrie \\ Qu'ils comprirent vraiment les vocables sonores \\ Et blessés à mourir surent qu'Eléonore \\ C'était ton nom Liberté Liberté chérie ${ }^{8}$}

C'est ce poème qui développe le plus le thème des croisades, par son titre et par l'isotopie correspondante, employée à double sens dans une écriture de contrebande. Dans son essai De l'exactitude historique en poésie, Aragon justifie par des finalités contemporaines les décalages qu'il fait subir à l'Histoire :

Je ne suis pas bien sûr que si on regardait attentivement «Le Paysan de Paris chante » ou «Absent de Paris », le second Empire, ou le temps de Philippe-le-Bel, ou le temps de Charles VI, en expliquerait parfaitement tous les écarts de langage. Je pense pourtant que le temps de Philippe Pétain s'accordait parfaitement des inexactitudes historiques qu'on pourrait accorder à ces poèmes ${ }^{9}$.

Les allusions historiques sont nombreuses dans cette poésie résistante et concernent aussi bien les rois fainéants ${ }^{10}$ que Philippe le Bel, Du Guesclin ${ }^{11}$, Charles VI ${ }^{12}$ ou Jeanne d'Arc ${ }^{13}$. Les expressions archaïques calquées sur l'ancien français sont elles-aussi présentes à plusieurs reprises et témoignent d'une connaissance approfondie des textes originaux ${ }^{14}$. La référence médiévale joue donc un rôle important dans la description de la période contemporaine.

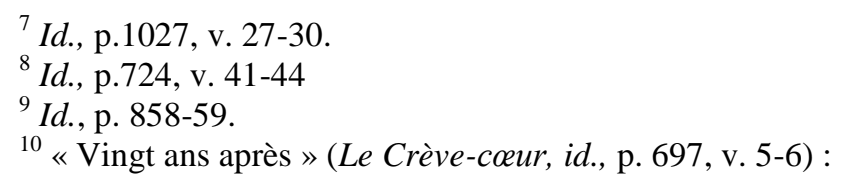

Jours carolingiens Nous sommes des rois lâches

Nos rêves se sont mis au pas mou de nos vaches

${ }^{11}$ «Plus belle que les larmes », Les Yeux d'Elsa (id., p. 789, v. 124).

${ }^{12}$ Dans «Absent de Paris » (Le Crève-coeur), le locuteur se compare à Charles VI (id., p. 884, IV, v. 15).

${ }^{13}$ Elle apparaît notamment dans «Richard II quarante » du Crève-cœur et dans « Poème de l'été 41 »d'En français dans le texte.

${ }^{14}$ Le poème «Chanson de récréance » (Les Yeux d'Elsa) fourmille de termes médiévaux : « récréance », signifiant abandon des valeurs chevaleresques au profit des plaisirs de l'amour, « reverdie » ou évocation du printemps, « merencolie », c'est-à-dire mélancolie et enfin « le latin des oiseaux », soit le chant des oiseaux (id., p. 779-80). On trouve aussi un «phénix navré », c'est-à-dire blessé, dans « Lancelot» du même recueil (id., p. 792, v. 82) et une lumière « entée », greffée, dans «Le paysan de Paris chante » (En français dans le texte, id., p. 874, v. 32). Enfin, deux expressions révèlent une syntaxe médiévale, le «boire d'amour » de la «Chanson de 


\section{Un Moyen Âge mythologique}

Pour Aragon, le Moyen Âge constitue de surcroît une source d'inspiration mythologique. Brocéliande, qui décrit la forêt enchantée de Merlin avec ses rites celtiques, est le seul recueil à faire explicitement référence au Moyen Âge dans son titre. Mais Aragon ne recrée le mythe de Brocéliande que pour le faire dévier vers une signification contemporaine : il transforme la réalité d'un pays occupé et divisé en une réalité seconde, susceptible d'enchantements. Dans ce contexte, le Moyen Âge sert à créer une distance par rapport au monde réel et à trancher sur les descriptions plus réalistes qui font partie intégrante des poèmes de résistance.

La forêt devient métaphore de la France. «De l'arbre où ce n'est pas Merlin qui est prisonnier », poème en terza rima (forme dantesque), reprend le mythe de Merlin dissimulé dans un arbre pour l'étendre à l'ensemble des résistants :
Ah combien de Merlins sous des pierres tombales
Et tous ces arbres sont des arbres enchantés
Tout à l'heure vous le verrez bien quand le bal
S'ouvrira quand brisant le cœur du bel été
L'étoile neigera le long des paraboles
Orage des héros orage souhaité ${ }^{15}$

La thématique forestière ${ }^{16}$ apparaît clairement comme le prétexte à un message résistant exposé au cœur du poème :

Chacun d'eux à l'appel de France répondant

Chacun d'eux a l'accent qu'il faut au sacrifice

La gloire n'eut jamais autant de prétendants ${ }^{17}$

L'idée d'une vie souterraine est mise en relief grâce au mythe de Merlin. Aragon ranime cet héritage médiéval pour recréer une cohésion nationale et proposer un modèle héroïque capable de pousser ses lecteurs vers la Résistance. Le mythe médiéval relève pleinement de la stratégie d'une poésie engagée ${ }^{18}$. La France contemporaine se transforme en un lieu propice aux légendes. La note (de la main de l'auteur) qui suit le poème «Légende de Gabriel Péri » dans La Diane française en témoigne :

Ce poème, écrit pour le second anniversaire de la mort de Gabriel Péri, publié illégalement, relève vraiment de la légende et non de l'histoire : en effet ce n'est pas à Ivry, mais à Suresnes et dans une tombe enregistrée et non pas dans la fosse commune que Péri est enterré. L'auteur, alors dans l'illégalité, n'a pourtant inventé aucun de ces détails [...] ; déjà la tradition orale avait porté jusqu'à lui moins de deux ans après la mort du martyr cette version

récréance » (Les Yeux d'Elsa, id., p. 779, v. 14) et « le monument du haut vouloir » du poème « Le jour se lève sur la fontaine des Innocents » (En français dans le texte, id., p. 902, v. 48).

${ }^{15}$ Id., p. 845 , v. 43-48

${ }^{16}$ De l'exactitude historique en poésie (Id., p. 865) vient corroborer cette interprétation :

Plus encore qu'en 1941, en 1942 la France tout entière ressemblait à Brocéliande. Dans la forêt, les sorciers de Vichy et les dragons de Germanie avaient donné à toutes les paroles une valeur incantatoire pervertie, rien ne s'appelait plus de son nom, et toute grandeur était avilie, toute vertu bafouée, persécutée. [... ] Et plus il avançait, ce temps, plus nombreux s'armaient les chevaliers sans nom, qui s'appelaient Roger ou Pierre, Daniel ou Jean, plus nombreux surgissaient les paladins dont les exploits, malgré les hommes d'armes et les bourreaux, et les ogres et les géants, se répétaient de bouche en bouche d'un bout à l'autre de la forêt de France ; si bien que ce fut une contagion extraordinaire de héros, une ivresse d'exploits, une réincarnation de la légende dans l'histoire.

${ }^{17} I d .$, p. 844 , v. $22-24$

${ }^{18}$ Dans De l'exactitude historique en poésie (Ibid.), Aragon souligne ses intentions en ce domaine : « Les mythes remis sur leurs pieds ont force non seulement de faire rêver, mais de faire agir ». 
déformée par quoi naît une légende aujourd'hui comme au temps de La Chanson de Roland, des Troubadours et des poèmes transmis de bouche en bouche à travers une France alors comme aujourd'hui dévastée et livrée aux soudards et aux chimères ${ }^{19}$.

Couvrant à la fois poésie, histoire et mythe, cette comparaison avec la période médiévale est tout à fait représentative des correspondances que le poète trace entre les époques.

\section{Le retour à des jeux sonores anciens}

Pendant la Résistance, Aragon revient massivement au vers pair compté, classicisme assez commun dans la poésie résistante qui vise avant tout la clarté du message. Son recours à des strophes et à des structures de rimes peu usitées depuis la seconde moitié du XVI ${ }^{\mathrm{e}}$ siècle est en revanche une originalité.

Ses rimes majoritairement riches sont marquées par des formes spécifiques issues des Grands Rhétoriqueurs : ce qu'il appelle « la rime complexe » (la rime équivoquée des Grands Rhétoriqueurs), la rime enjambée (rime incomplète qui trouve son complément dans l'adjonction de la consonne initiale du vers suivant), la rime brisée (qui fait rimer les vers par la césure), la rime batelée (qui fait rimer la fin du vers avec la fin de l'hémistiche suivant), la rime annexée (qui reprend la syllabe de la rime au début du vers suivant) et enfin le vers léonin (ou rime interne).

Quant au jeu sur les ambiguités métriques, il reprend la tradition occitane. Une double structure strophique rend certains poèmes lisibles comme des poèmes en alexandrins ou comme des poèmes en vers plus courts, octosyllabes ou hexasyllabes, rimant entre eux. L'exemple le plus frappant en est la « Plainte pour le grand descort de France » (Les Yeux d'Elsa), où structures de l'alexandrin et de l'hexasyllabe se chevauchent, dans des vers biocatz (à gauche le poème tel qu'il est présenté, à droite son redécoupage possible) :

S'il se pouvait un chœur de violes voilées S'il se pouvait un cœur que rien n'aurait vieilli Pour dire le descort et l'amour du pays S'il se pouvait encore une nuit étoilée S'il se pouvait encore ${ }^{20}$
S'il se pouvait un chœur

De violes voilées

S'il se pouvait un cœur

Que rien n'aurait vieilli

Pour dire le descort

Et l'amour du pays

S'il se pouvait encore

Une nuit étoilée

S'il se pouvait encore

Jeu métrique, cette structure est aussi à mettre en rapport avec le sens caché du poème, éclatant à la dernière strophe (Aragon y évoque les morts du mois de mai, il semble y désigner ceux de mai 1940 mais la mention d'une muraille doit orienter la lecture vers la référence à mai 1871 et à la Commune). Cette structure sous la structure illustre à la fois l'idée d'emprisonnement et la présence sous-jacente d'un espoir,

Dans le titre du poème, Aragon emploie le mot ancien de descort, signifiant à la fois désunion et discorde, ce qui lui permet de jouer sur un double sens littéral et figuré. Par ailleurs, ce terme renvoie aussi à un genre poétique de la lyrique occitane. Poème désordonné, marquant la colère du troubadour face à des sentiments non partagés, le descort était composé de cinq à dix strophes toutes différentes par le nombre de leurs vers, leurs rimes, leurs mètres et parfois leur thème. Chaque strophe reposait sur une mélodie différente et Aragon l'illustre en changeant ici de tonalité selon les strophes.

Les jeux sonores ne se limitent pas à la rime, Aragon les introduit au cœur même de ses vers. Dans «La Constellation » (Les Yeux d'Elsa), le locuteur fait un retour spéculaire sur la création du poème :

${ }^{19}$ Id., p.1026.

${ }^{20}$ Id., p. 777, v.1-5. Aragon donne lui-même la construction de ce poème dans Arma virumque cano, la préface des Yeux d'Elsa (id., p. 754). 


\title{
Je tresserai mes vers de verre et de verveine \\ Je tisserai ma rime au métier de la fée \\ Et trouvère du vent je verserai la vaine Avoine verte de mes veines \\ Pour récolter la strophe et t'offrir ce trophée ${ }^{21}$
}

Aragon est ici très proche des vers senés, dont tous les mots commencent par la même consonne, employés aussi bien par les troubadours que par les Grands Rhétoriqueurs. Cet effet sonore apparaît précisément au moment où le locuteur affirme une détermination lyrique et se présente comme un trouvère. La célébration de la femme aimée, appuyée par des effets sonores d'origine médiévale, vient soutenir le message résistant, amour d'Elsa et amour de la France étant liés.

Outre des formes métriques, Aragon reprend des formes poétiques médiévales comme la canso ou la reverdie, issues de la lyrique occitane, mais aussi des formes fixes du Moyen Âge tardif, comme la ballade et le rondeau. "Richard II Quarante » (Le Crève-cœur) est une ballade sans envoi, en octosyllabes qui décrit la défaite française et la situation politique du pays après l'Armistice :

\author{
Le cœur peut s'arrêter de battre \\ Le sang peut couler sans chaleur \\ Deux et deux ne fassent plus quatre \\ Au Pigeon-Vole des voleurs \\ Je reste roi de mes douleurs ${ }^{22}$
}

Le jeu du refrain «Je reste roi de mes douleurs » permet une insistance lyrique et, par sa répétition systématique, marque la persistance de la souffrance. Il est corroboré par la série d'adynata qui par contraste soulignent la permanence. Ainsi Aragon exploite-t-il la forme spécifique de la ballade pour faire passer un message de ténacité, de détermination. Le fait que cette forme fixe soit réinsérée dans une poésie résistante n'est d'ailleurs pas sans rapport avec ses vertus mnémotechniques : c'est le support idéal d'un discours idéologique, lui-même souvent marqué par des phénomènes de réitération.

L'esthétique médiévale de la ballade est pleinement mise au service de l'expression engagée, ses particularités formelles propres viennent soutenir le message résistant.

\section{Le lyrisme}

De fait, entre 1940 et 1945, la poésie d'Aragon prend une tonalité très lyrique. L'évocation d'Elsa s'opère par l'entremise de la lyrique occitane qui fournit le modèle du chant d'amour, la canso. "Six tapisseries inachevées » (La Diane française) est le poème qui fait entrer en correspondance lyrisme et engagement de la façon la plus nette :

J'ai rencontré ma Dame au bord de l'eau

Ma Dame est France et moi son Lancelot ${ }^{23}$.

Ces vers simples dressent une équivalence entre la dame de la tradition courtoise et la figure allégorique de la France. On retrouve semblable effet dans «Lancelot » (Les Yeux d'Elsa) où l'amour du locuteur-chevalier s'adresse au pays tout entier :

En étrange pays dans mon pays lui-même

Je sais bien ce que c'est qu'un amour malheureux ${ }^{24}$.

\footnotetext{
${ }^{21} I d .$, p. 796, v. 6-10.

${ }^{22}$ Id., p.720, v. 11-15

${ }^{23}$ Id., p. 997 , v. 11-12.

${ }^{24} I d$., p. 792, v. 87-88.
} 
Aragon reprend ici une tradition de la lyrique occitane, illustrée notamment par Peire Vidal ; l'une de ses cansos superpose amour du pays dont il est exilé (en l'occurrence la Provence) et amour d'une femme :

\author{
Ab l'aler tir vas me l'aire \\ Qu'ieu sent venir de Proensa : \\ Tot quant es de lai m'agensa \\ Si que, quan n'aug ben retraire \\ Eu m'o escot en rizen \\ E'n deman per un mot cen : \\ Tant m'es bel quan n'aug ben dire ${ }^{25}$
}

Pour Aragon, l'amour est une valeur opposée à l'inhumanité de la guerre; facteur de civilisation, il participe du combat humaniste de la Résistance. On retrouve semblable conception chez le troubadour Peire Cardenal, poète de la résistance occitane, auteur de sirventes politiques, qui, lors de la croisade albigeoise, considérait la fin' amor comme l'emblème d'une civilisation que les Français et l'Eglise voulaient détruire.

Dans La Leçon de Ribérac, Aragon défend sa relecture de la littérature médiévale en en expliquant l'adéquation par rapport à la Résistance :

On me dira qu'il y a des tâches plus pressantes que l'étude de Chrétien de Troyes et des poètes de notre douzième siècle, et j'en conviens sans peine. Les mêmes gens me le diront qui se sont peut-être alarmés de me voir plus haut faire l'éloge de Maître Arnaud Daniel et de son "clus trover", de l'art fermé [... ]. Sans doute de cet héroïsme d'aujourd'hui, de cette fidélité profonde, y a-t-il des milliers d'exemples vivants qui me dispenseraient de Perceval ou de Tristan ${ }^{26}$. Mais peut-on aujourd'hui en parler ? Assurément pas. C'est eux que je salue en Perceval, le Chevalier vermeil. Et pour ceux qui me diront : "Mais l'art fermé, voyons ! [... ]" je leur rappellerai que $[\ldots][\mathrm{le}]$ "clus trover" permettait aux poètes de chanter leurs Dames en présence même de leur Seigneur ${ }^{27}$.

Le lien entre inspiration médiévale et Résistance est ainsi établi en ce qui concerne l'héroïsme et la circulation des mots à couvert, la « contrebande » selon le terme d'Aragon, qui passe justement par l'exemple du lyrisme. Le lyrisme est donc thématiquement et formellement lié à la pratique d'une écriture engagée qui veut défendre une série de valeurs issues de la courtoisie.

Le poème « Pour un chant national » (Les Yeux d'Elsa) dresse une comparaison explicite entre l'époque contemporaine et celle de Bertran de Born, tout en élevant ce troubadour au rang de modèle en termes d'engagement poétique :

\title{
Mais pourtant lorsque vint la grêle \\ On entendit chanter Bertrand ${ }^{28}$
}

Bertrand de Born est d'ailleurs passé de la poésie amoureuse à la poésie engagée. Le célèbre poème où il retourne le genre de la reverdie en témoigne : au lieu de célébrer le printemps comme saison amoureuse, topos et même fondement du genre, il en fait la saison de la guerre :

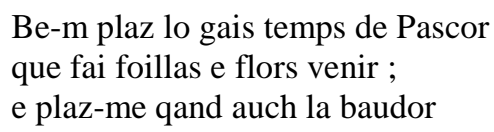

${ }^{25}$ Peire Vidal, Poésies, éd. et trad. Joseph Anglade, Paris, Champion, 1913, p. 60 : Avec mon haleine, j'aspire l'air que je sens venir de Provence ; tout ce qui touche à cette contrée me plait ; aussi quand j'en entends dire du bien, je suis si heureux que j'écoute en souriant et que pour un mot j'en demande cent.

${ }^{26}$ Dans l'œuvre d'Aragon, la figure de Tristan apparaît dans les poèmes « Lancelot » et « Chanson de récréance » des Yeux d'Elsa.

${ }^{27} \mathrm{OPC}$, p. 820-21.

${ }^{28} I d$., p. 782, v. 25-26. « Pour un chant national » reprend aussi une forme empruntée à un autre troubadour. En effet, la clausule en prose qui fait un retour sur le locuteur et la situation présente, s'inspire de la forme remarquable d'un poème célèbre de Raimbaut d'Orange, «Un ballot de folie ». 
dels auzels, que fant retentir

lor chan per lo boscatge ;

e plaz-me quand vei per los pratz

tendas e pavaillon fermatz ;

et ai gran alegratge

qand vei per campaignas rengatz

cavalliers e cavals armatz ${ }^{29}$

Le renouveau formel dont bénéficie la poésie d'Aragon à cette époque doit beaucoup à sa lecture des troubadours. Paradoxalement, le chant amoureux vient s'insérer dans la poésie résistante et la soutenir, l'innerver; il reste néanmoins que l'évocation du Moyen Âge crée toujours un décalage.

\section{L'effet Moyen Âge dans une poésie de la Résistance : un jeu sur le décalage}

Aragon joue en fait sur une mise en scène du Moyen Âge dont l'apparition est soulignée pour produire certains effets, notamment dans les poèmes satiriques. Dans « Absent de Paris » (En étrange pays dans mon pays lui-même), le locuteur passe plusieurs strophes à décrire une situation de conflit interne au présent, avec des vers très marqués par une tonalité résistante ; il revient seulement ensuite à un Moyen Âge tout juste esquissé, et le texte est alors au passé :

Votre Dies irce semblait incendiaire

C'est vers l'an treize cents que ceci se passait

Au psaume très ancien s'ajoutent des versets

Sur les lèvres de feu de nos héros d'hier

On entendait les loups près du Louvre et ce n'est

Qu'au galop qu'on fuyait les hommes de gabelle

Dans l'étrange Paris de Philippe-le-Bel

Le Roi même faisait de la fausse monnaie ${ }^{30}$

Le vers qui indique la date des faits paraît presque comique dans son désir de précision historique. Aragon dévoile les ficelles à son lecteur, puisqu'il est immédiatement fait mention d'un temps plus récent avec les « héros d'hier ». Toute la description des luttes présentes s'insère entre deux mentions ambivalentes du Paris médiéval. Sont surtout mis en relief (au tout début et à la fin) les deux vers les plus explicitement accusateurs, ceux où le prénom Philippe apparaît, avec son double sens historique.

L'effet médiéval est donc souligné dans un but satirique. C'est aussi le cas du poème « Les Neiges de Sigmaringen » : parodie de la «Ballade des dames du temps jadis » de Villon, il a pour refrain « Où est Darquier de Pellepoix », que vient encadrer au début et à la fin « Où est ma cinquième colonne $»^{31}$. Ce poème, dont le titre souligne d'emblée la forme parodique, constitue une violente satire de tous les collaborationnistes exilés, avec une énumération de noms, comme dans l'hypotexte.

La thématique médiévale permet donc de faire passer de manière indirecte un message politique; ce système trouve son achèvement dans ce mode de propagande qu'Aragon appelle « contrebande ».

${ }^{29}$ Le Seigneur-Troubadour d'Hautefort, L'Euvre de Bertran de Born, éd. et trad. Gérard Gouiron, Aix-enProvence, Presses de l'Université de Provence, 1987, p. 519 : Je trouve un grand plaisir dans le joyeux printemps de la saison nouvelle qui fait pousser feuilles et fleurs ; et j'ai plaisir à entendre la gaîté des oiseaux qui font retentir leur chant par le bocage ; et j'ai plaisir à voir dressés par les prés tentes et pavillons et je ressens une grande allégresse quand je vois en lignes par les champs cavaliers armés sur leurs chevaux.

${ }^{30} I d$., p. 881-82, partie II du poème, v. 45-52. Pour financer ses guerres, Philippe le Bel changea la valeur du cours de la monnaie, ce qui lui valut la réputation de faux-monnayeur.

${ }^{31}$ Id., p. 966-967. 


\title{
La contrebande
}

Façon d'exprimer le discours résistant à mots couverts, la contrebande, donne lieu à des sousentendus, allusions et métaphores qui trouvent dans le Moyen Âge un comparant. Mais cette forme de propagande n'est efficace que si elle parvient à faire comprendre son double message, ce qui implique des mises en relief spécifiques. À la fin de «Ce que dit Elsa » («Cantique à Elsa », Les Yeux d'Elsa) Aragon fait un retour réflexif sur cette pratique du double sens:

\author{
Tu me dis Si tu veux que je t'aime et je t'aime \\ Il faut que ce portrait que de moi tu peindras \\ Ait comme un ver vivant au fond du chrysanthème \\ Un thème caché dans son thème \\ Et marie à l'amour le soleil qui viendra ${ }^{32}$
}

Créant un décalage, la référence médiévale suscite une évocation indirecte de la réalité qui se révèle précieuse dans une littérature censurée ; elle devient alors plus efficace qu'une expression directe. Le détour par le Moyen Âge relève donc pleinement d'une stratégie résistante.

Dans un Entretien avec Francis Seguin en 1969, Aragon s'étend sur la dialectique de la contrebande, prise entre codification et clarté :

[... ] j'avais pensé qu'il fallait une poésie relativement claire, mais qui ne le soit pas au point d'attirer l'attention de la censure. En somme, une poésie de contrebande, mais qui ne poserait pas de problème de forme. Une poésie qui, pour les gens, serait directement appréhensible parce qu'elle relèverait d'une certaine tradition à quoi se reconnaîtrait la masse des Français ${ }^{33}$.

Fréquemment, jeux stylistiques viennent donner un indice de cette contrebande; le déguisement du message est lui-même mis en relief, avec un effet d'insistance qui équivaut à un discours quasi explicite. Dans «Les Croisés » (Le Crève-cœeur), Aragon souligne le prêche de Pierre l'Ermite par un style direct qu'il insère dans le poème : «Ah quand ils entendaient dire La Terre Sainte ${ }^{34} \gg$ et 《Quand le prédicateur disait Jérusalem ${ }^{35}$ ». Le texte mime le prêche réel et met en valeur des toponymes derrière lesquels plane l'ombre d'autres lieux à défendre.

Dans le contexte du Crève-cœur, il est d'autant moins difficile d'entr'apercevoir ici un écho à la France que l'analogie devient claire dès le huitième quatrain, avec la mention « d'une autre défaite / À laquelle il n'y a pas de deleatur ${ }^{36}$ ». La symbolique historique de ces « croisés » cède la place à une signification politique contemporaine.

La contrebande renforce ainsi l'artificialité du Moyen Âge dans une œuvre qui en est cependant profondément inspirée. En ramenant inéluctablement le lecteur à la nécessité de l'action, la mise en relief des effets sert avant tout la perspective résistante.

\section{Le miroir de l'épopée}

Outre le lyrisme, l'épopée constitue aussi un modèle engagé pour la poésie résistante d'Aragon ; « Du poète à son parti » (La Diane française) le dit :

Mon parti m'a rendu le sens de l'épopée

Je vois Jeanne filer Roland sonne le cor

C'est le temps des héros qui renaît au Vercors

Les plus simples des mots font le bruit des épées

\footnotetext{
${ }^{32} I d .$, p. 798 , v. $1-5$.

${ }^{33}$ Entretien avec Francis Seguin, in Euvres Poétiques Complètes, Monaco, Alphée, 1979, tome XV, p. 67.

${ }^{34}$ OPC, p. 723, v. 21.

${ }^{35} \mathrm{Ibid}$, v. 27

${ }^{36}$ Ibid., v. 31-32.
} 
Aragon inscrit les combats contemporains dans la filiation de la geste médiévale ; il souligne le rôle du langage dans ce renouveau épique qui semble toucher la France. Les autres dons du parti communiste dans ce poème sont « [les] yeux et [la] mémoire ${ }^{38}$ » et « les couleurs de la France ${ }^{39}$ »; l'épopée constitue donc le troisième point du patriotisme après la mémoire historique et les symboles nationaux. Sa mise en avant correspond à une idéalisation de la nation dont cette forme littéraire devient l'emblème. La préface aux Trente-trois sonnets composés au secret de Jean Noir (Jean Cassou) est exemplaire de ce phénomène, qu'elle reproduit dans sa propre textualité, puisque le texte critique devient lui-même épique :

Des prisons qui sont les sanctuaires de la Patrie monte la nouvelle chanson de Roland, d'un Roland aux mille et mille têtes, et sa voix ne se brisera pas à souffler l'olifant. Car déjà le monde entier l'entend, frémit, et va répondre. ${ }^{40}$

L'aspect incantatoire, prophétique, de la fin de ce paragraphe voit Aragon modeler sa période sur le souffle épique. Cette introduction à l'œuvre d'un autre poète résistant prend donc l'épopée comme référence pour redonner vie à une image de la nation, alors même que l'œuvre de Cassou est un simple recueil de sonnets.

Le fait qu'Aragon utilise cette métaphore prouve que son interprétation du monde réel se modèle sur des éléments médiévaux, dont il accentue même la présence, parce qu'il y voit les germes de la nation à reconstruire ${ }^{41}$. Lyrique ou épique, la littérature médiévale est donc mise à contribution dans l'édification d'une idéologie nationale combattant celle de Vichy ${ }^{42}$.

Le jeu médiéval d'Aragon entre 1940 et 1945 s'avère ainsi complexe : on peut observer une imprégnation générale de son œuvre par des thèmes ou formes issus du Moyen Âge, mais ce dernier est aussi une image, le prétexte à une poésie résistante qui s'affirme indirectement. Le renouveau formel qui guide toute cette production de guerre est amplement redevable aux formes médiévales, dans la mesure où Aragon reprend jeux sonores et formes poétiques des troubadours et de leurs successeurs. Quant au lyrisme qui marque fondamentalement cette période de l'œuvre aragonienne, il suit le modèle courtois qu'il ne cesse de revendiquer.

${ }^{37}$ Id., p. 1031, v. 6-10

${ }^{38}$ Id., p. 1031, v. 5.

${ }^{39}$ Ibid., v. 11

${ }^{40}$ Louis Aragon, L'Euvre poétique, tome X, 1943-1945, Paris, Idées et Éditions, Livre Club Diderot, 1979, p. 374.

${ }^{41}$ La référence médiévale est encore présente après guerre, comme en témoignent ces vers de la « Comptine du quai aux fleurs » du Nouveau Crève-cœur (OPC, p. 1063, v. 5-8) :

C'est ce peuple qui commence

Son histoire à Roncevaux

Roland l'ancienne romance

Et Fabien le chant nouveau

42 La méditation d'Aragon sur le lyrisme s'alimente d'une rêverie historique dont il décrit bien lui-même la fonction dans La Leçon de Ribérac (Louis Aragon, OPC, p. 809-10): Non, ce qui me retenait dans l'image d'Arnaud Daniel, ce n'était pas seulement l'art fermé, cette incroyable invention de règles nouvelles, de disciplines que le poète s'impose et fait varier à chaque poème, [... ] c'était que, dans un temps où mon pays était divisé, et par la langue, et dans sa terre, où il y avait un roi de Paris, et un roi d'Angleterre qui tenait la moitié de la France, et dans le Nord un comte de Flandre, dans l'Est un comte de Champagne, dans un temps où mon pays était encore épuisé par les folles saignées des Croisades, qui seules remettaient d'accord ces princes ennemis contre les ennemis de l'Est lointain, il se soit développé une poésie qui porta plus loin et plus haut que les étendards de ces princes la grandeur française, et fit naître dans l'Italie de Virgile et d'Ovide une gloire, une grandeur nouvelles, qui se réclament de la France. J'étais saisi de cette idée, quand tout paraissait perdu, elle venait me rendre le courage et la confiance en nos destinées, et c'est de quoi je resterai à jamais reconnaissant à Maître Arnaud Daniel. 
Conception courtoise et sens épique sont donc présentés comme des modèles dignes de guider le mouvement résistant. Le Moyen Âge est aussi mis en scène, instrumentalisé, dans une optique résistante, la contrebande constituant une arme efficace contre la censure ainsi qu'un fonds intéressant de double sens et de mystère : dans «La romance du temps qu'il fait », poème du Crève-cœur ${ }^{43}$, Aragon précise d'ailleurs : « Je garde le secret du jeu».

Armelle Leclercq CEMA, Université Paris III

\footnotetext{
${ }^{43} I d .$, p. 712 , v. 35.
} 
Bibliographie

Euvres littéraires

Euvres de Louis Aragon

Le Crève-cour, Paris, Gallimard, 1941

Les Yeux d'Elsa, Neuchâtel, Cahiers du Rhône, 1942

Brocéliande, Neuchâtel, La Baconnière, 1942

Le Musée Grévin, Paris, Éditions de Minuit, 1943

La Diane française, Paris, Seghers, 1944.

Euvres Poétiques Complètes, Monaco, Alphée, 1979

L'Euvre poétique, Paris, Idées et Éditions, Livre Club Diderot, 1979

Euvres poétiques complètes, sous la direction d'Olivier Barbarant, Bibliothèque de la Pléiade, Paris, Gallimard, 2007

Euvres de troubadours

Peire Vidal, Poésies, éd. Joseph Anglade, Paris, Champion, 1913

Le Seigneur-Troubadour d'Hautefort, L'Euvre de Bertran de Born, éd. Gérard Gouiron, Aixen-Provence, Presses de l’Université de Provence, 1987

Etudes critiques

Pierre Daix, Aragon, une vie à changer, Paris, Flammarion, 1994

Élodie Burle et Valérie Naudet, « Nager à contre-courant (une approche du sujet lyrique entre mesure et démesure à partir de quelques poèmes d'Arnaut Daniel et Louis Aragon) », Revue des langues romanes, t. CX, 2006, $\mathrm{n}^{\circ} 2$, p. 507-525

Philippe Gardy, "Aragon lecteur des troubadours : emprunt et déplacement d'un mythe », Mythes, Images, Représentations, Trames, sous la direction de Jean-Marie Grassin, Limoges, Didier, p. $269-277$

Roger Lassalle, "La poésie médiévale dans l'œuvre critique et l'œuvre poétique d'Aragon », L'Image du Moyen Âge dans la littérature française de la Renaissance au XX $X^{e}$ siècle, Poitiers, La Licorne, 1982, t. 1, p. 195-211.

Mots-clefs

Auteurs: Louis Aragon, Arnaut Daniel, Peire Vidal, Bertran de Born, Peire Cardenal, Raimbaut d'Orange, Blondel de Nesle, François Villon, Alain Borne, Jean Cassou

Euvres : Le Crève-cœur, Les Yeux d'Elsa, Brocéliande, En Français dans le texte, Le Musée Grévin, La Diane Française, La Conjonction Et, La Leçon de Ribérac, Les Poissons noirs ou de la réalité en poésie

Notions : résistance, action, courtoisie, rime, croisade, descort, canso, contrebande, reverdie, trobar clos, parti communiste 